\title{
A metaphorical and visual language for querying an existing relational database
}

\author{
Bruno BÉLIÈRES (1)(2) and Claude TRÉPIED (3) \\ (1) SLIGOS, 641 Avenue du Grain d'Or, 41350 Vineuil,
} France

(2) Laboratoire d'Informatique, Université de Tours E3i, 64 Avenue Jean Portalis, 37200 Tours, France belieres@univ-tours.fr

(3) Laboratoire d'Ingénierie des Systèmes d'Information, Université Lyon I, INSA de Lyon, Bat.404, 69621 Villeurbanne Cedex, France trepied@univ-tours.fr

\begin{abstract}
Metaphors have been proved useful in facilitating learning and using an operating system or various other tools. The purpose of this paper is to show through the VISTA language, the benefits of using metaphors for the reverse engineering of relational databases. VISTA is a visual query language associated with an objectoriented semantic data model. VISTA uses metaphors to represent the data model concepts and the language operators. It provides a meaningful query environment for end users. The user is set in a room and querying consists of direct and natural manipulations on objects of this room. We show how VISTA proposes a unified solution to problems such as set comparison and combination, explicit joins, quantification, graphic congestion while seeking at the same time easy query formulation. We also report our experience with an industrial example.
\end{abstract}

\section{Keywords}

Visual query language, end user, metaphor, semantic visualization, object-oriented semantic data model, database reverse engineering, industrial application. 


\section{INTRODUCTION}

The reuse of existing relational databases is an interesting challenge nowadays (Premerlani and Blaha, 1994), especially for economical reasons. Many computerized activities are organized around a relational database, but the current query languages (e.g., SQL) are not adapted to end users. In this paper, we will focus on this final aspect of the semantic elicitation process when we obtain a database schema and we want it to be understandable and useful for an end user. Providing end user with a better suited language would enable him to query the database. That is typically the case in our banking application where we want to supply team managers with an adequate tool for banking activity analysis.

In order to query a database, the end user must know its schema. Furthermore, he uses a language to express his queries and this language refers to the concepts of the data model. So it is necessary, in order to build an interface that enables the end user to express his queries, to follow an approach integrating three notions: the definition (or the choice) of a conceptual data model, an end user-oriented visualization of the data model concepts, and the definition of query mechanisms, i.e. the language.

Various languages based on the use of graphic capabilities for improving database querying have been discussed in the literature (Chang and al., 1992) (Batini and al., 1993) (Dennebouy and al., 1995). We distinguish between tabular languages, graphical languages and visual languages (Aufaure-Portier and Trépied, 1996). We must also mention the hypermedia approach (Boursier and Mainguenaud, 1992) which enables the user to browse a database by following semantic or structural links between informational nodes (e.g., text, picture, map and sound). However, this approach cannot be considered as a query language because only predefined links can be used.

Tabular languages (e.g., OOQBE (Staes and al., 1991) and VQL (Vadaparty and al., 1993)) use forms or tables to convey information; the user fills them in to build his queries. These languages frequently need joins because of information scattered in different tables or forms. However, this operation is difficult and may lead to mistakes.

Concerning graphical languages (e.g., CANDID (Schneider and Trépied, 1989) and VQL (Mohan and Kashyap, 1993)), the schema is displayed as a graph with nodes representing object types and arcs pointing out relationships between these object types. Depending on the system, the user specifies his queries either by setting up conditions directly on the schema or, by building them in a separate window using graphical primitives. Nonetheless, representations of both the schema and the query remain abstract: the graphical conventions and their underlying concepts are difficult to comprehend.

The main feature of visual languages (e.g., Sketch! (Meyer, 1992) and Cigales (Calcinelli and Mainguenaud, 1994)) is the use of metaphors to show data model concepts or language operators. The user specifies his queries by manipulating objects of the application. A metaphor establishes an analogy between unknown 
concepts and familiar ones in order to make them understandable. Metaphors appear to be useful in the field of databases for schema visualization and query formulation (Mark, 1992) by translating abstract concepts of database querying to end users.

Nevertheless, problems remain. In spite of the fact that some operators are still difficult to express (e.g., set comparison operators, universal quantifier, explicit joins and class combination operators), the problem of their expression is seldom tackled in the literature. These languages inherently need space, and the limitations imposed by the screen may hinder or prevent the expression of some queries. The expressive power of a language is frequently limited by the weak semantic expressiveness of the data model. Moreover, the suggested visualizations do not always cover all aspects of the schema (e.g., the specialization-generalization relationship); as a result, end users may not be able to grasp all the semantics of an application.

The VISTA language proposes a unified solution to these problems. It is associated with an object-oriented semantic data model. Moreover the visualization model, which visualizes the data model concepts, helps end users to understand the schema. We also introduce the query room metaphor and a set of associated metaphors (e.g., scales).

This paper covers the following sections. Section 2 briefly describes the semantic data model and its visualization model. Section 3 introduces the metaphorical and visual query language: a general overview of the user interface and three metaphors (scales, lasso and shelf) are presented. Section 4 presents the general architecture of the system. Finally, Section 5 contains our conclusions and directions for future work.

\section{THE DATA MODEL AND THE VISUALIZATION MODEL}

The data model is an object-oriented semantic data model inspired by CANDID (Schneider and Trépied, 1989). In designing a visual query language for end users, we chose to limit the data model to simple abstraction mechanisms. Therefore we adopted a philosophy of modeling by attributes and not by type constructors (Hull and King, 1987).

A visualization model is associated with the data model. It maps abstractions of the data model into visual representations: its goal is to make the database schema easier for end users to understand and acquire.

This paper is illustrated with an example database in the banking area, although the language is not restricted to this area. This database deals with banks, credit cards and people. A bank manages credit cards; a person is client of one or more banks and own one or more credit cards.

A simplified representation of the database schema in CANDID style is given in Figure 1. 


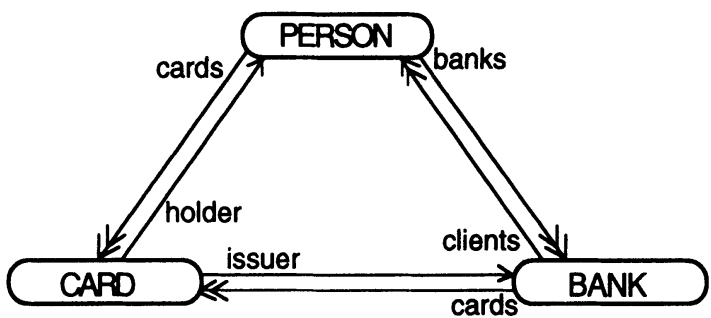

$\longleftarrow$ single-valued attribute
$\longleftarrow$ multivalued attribute

Figure 1 The example database.

We now present each concept of the data model with its visual representation as seen by end user.

An object is a part of the real world which is identifiable and which can be handled as a whole independently from other objects. It is identified by a unique reference called the object identifier. The state of an object corresponds to the collection of values taken by its attributes.

An object type is a definition by intention; we differentiate mediatic object types (MOTs) from abstract object types (AOTs). MOTs are used in terms of database input/output; we distinguish number, string, date, time, image and sound MOTs. An MOT is displayed as a rectangle containing its name (Figure 2).

\section{NAME}

Figure 2 An MOT.

AOTs are used to define objects that cannot be placed on a media (e.g., displayed on the screen) but that can only be described. They can be objects existing in a material form such as a card or in an immaterial form such as a telephone call. An AOT is represented by an icon composed of its image and name (Figure 3).

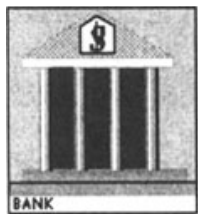

Figure 3 An AOT.

The icon is a metaphor; its components (an image and a label) suggest a concept which can be an element of the schema or an operation (Fujii and Korfhage, 1991) (Trépied, 1995). The fact that an icon may have a label enables to be precise on the 
meaning of the image and avoids problems of ambiguity. By extension, we say that an object has the same representation as its type. A class contains objects sharing the same object type and constitutes a definition by extension.

An attribute defines a relationship between a source object type and a target object type. It enables the designer to describe an aspect of an object through a semantic link with another object. There are no restrictions on types: the source or target object types may be MOTs or AOTs. An attribute is characterized by a name that can be explicit, when it is specified by the schema designer, or implicit. In this case, the attribute name is that of the target type. The source object is placed in a frame and its attributes are displayed in the panels bordering the frame (Figure 4). The benefits of this representation are to group all its attributes around an object type and to make implicit joins easier to formulate.

The attributes whose target type is an MOT are represented in the side panel by a rectangle containing their names. The attributes whose target type is an AOT are represented by an icon. Its image is the one of the target AOT and its label is the name of the attribute. These icons are put together in the lower panel. If the available space in a panel is not sufficient to contain all the attributes, the user has access to the non-visualized attributes by using scroll arrows.

An attribute is single-valued if, for each object of the source type, it corresponds to a maximum of one object of the target type. An attribute is multivalued if, for an object of the source type, it may correspond to several objects of the target type. A sequence of attributes forms a path. The path is said to be single-valued if it is composed of only single-valued attributes; otherwise the path is said to be multivalued.

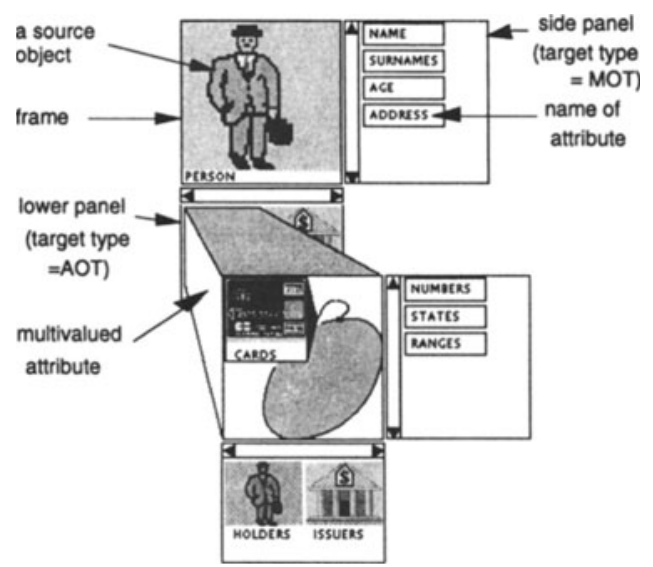

Figure 4 Attributes.

The selection of an attribute results in the display of the target object(s) and its (their) attributes. This functionality can be seen as a kind of zoom on parts of the schema and enables the language to cope with complex attributes. This display takes into account the path's valuation between the source type and the target type 
of the given attribute. For a multivalued attribute, a lasso will represent the set of target objects.

Attributes as they are defined in VISTA have a high semantic expressiveness and can simulate more complex abstraction mechanisms such as set constructors or tuple constructors (Hull and King, 1987).

\section{THE METAPHORICAL AND VISUAL QUERY LANGUAGE}

\subsection{The user interface}

Direct manipulation is a metaphor that replaces keyboard-entered commands with actions (e.g., drag and drop) on objects of the interface. Direct manipulation in VISTA goes beyond the level found in WIMP (Window-Icon-Menu-Pointing) interfaces (Egenhofer and Bruns, 1995): actions are done on real-world objects (e.g., scales and stickers) rather than on simple buttons and menus. This enhances the quality of interaction and adaptation to the user because he is placed in a concrete world and acquires a sensorimotor representation (Roby-Bramy, 1993) of the different interface components. He gains a knowledge by seeing them and by the different manipulations he can do on them.

The interface constitutes the working environment which enables the user to express his queries. The metaphor used is a room called the query room (Figure 5).

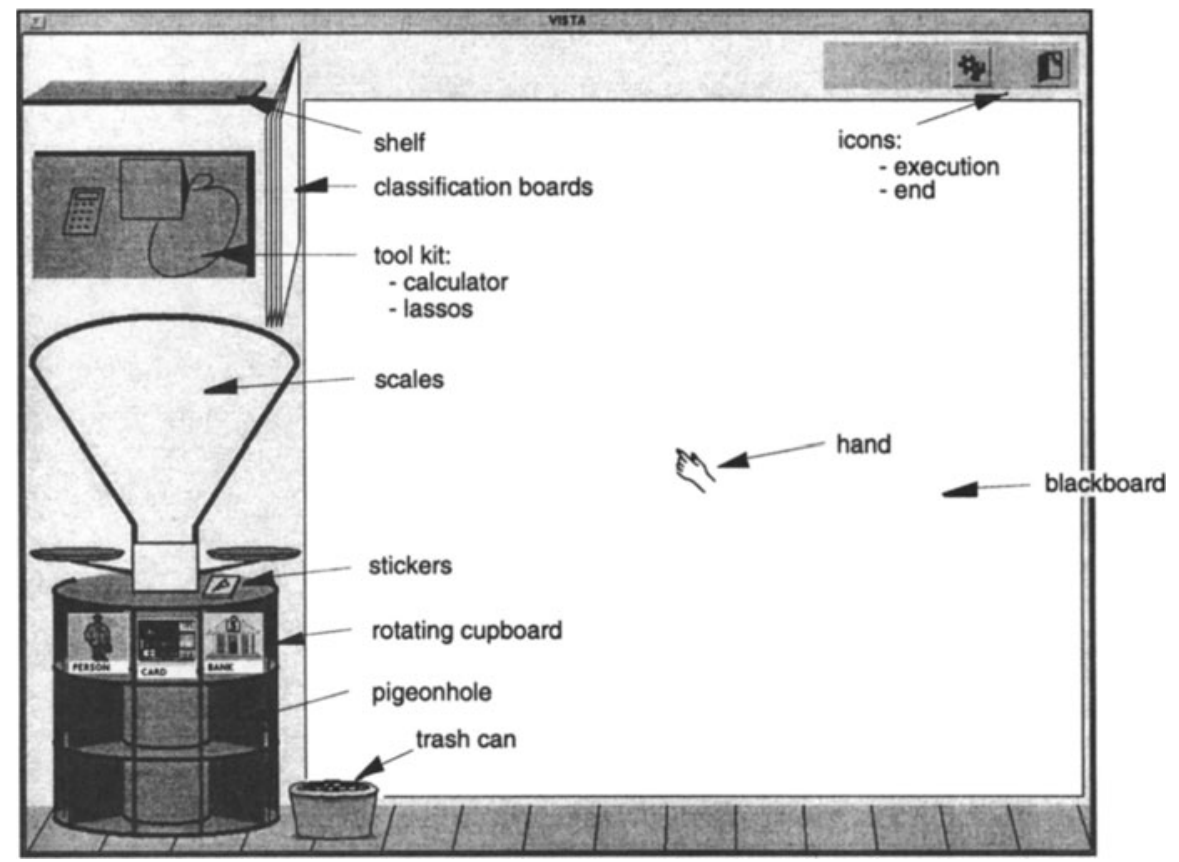

Figure 5 VISTA, the query room. 
In this room, a blackboard is displayed and the user describes his queries on it. The pointing device is a hand; the hand is already used in other user interfaces and enhances the idea of direct manipulation. The rotating cupboard contains icons of object types (only AOTs) in its pigeonholes.

VISTA is a declarative visual language; it is based on a query-by-example philosophy. The expression of a query consists in describing one or more example objects from one or more object types that the user brings to the blackboard.

The scales which are used to specify comparison conditions and stickers which are used to mark attributes to be displayed in the result are the basic tools when building a query. The other tools are used to construct more complex queries.

A tool kit contains different tools: a calculator to specify functions and lassos to switch from the object notion to the set notion, in particular for class combinations. Classification boards describe the various hierarchies of specialization-generalization. The shelf enables the user to compose incrementally a query by stocking subqueries on it. Empty pigeonholes in the rotating cupboard are employed to store queries within the database, thus allowing their reuse in later sessions. Finally, a trash can is used to remove elements of the blackboard. As it is common in other interfaces, two icons located at the top right are provided to run the query and to leave the application. The former is represented as gears and the latter as an exit door.

In order to avoid repeating for each query the image of the interface in its entirety, we limit the figures to the snapshots of the schema and interface elements involved in the query.

\subsection{The scales metaphor}

The scales metaphor allows the user to express explicit joins by direct manipulation in an intuitive way (Bélières and Trépied, 1995). The scales metaphor guides the user while he specifies a comparison condition.

To formulate such a condition, the scales are available in the query room. So, the user takes a first operand (an object or a class) and brings it to one of the pans of the scales. Then, he must specify on the other pan the second operand either by using the keyboard (in the case of a constant from a mediatic object type) or, by bringing its rectangle or icon. It is worth noting that both operands may be variables. Colored coding of the operands, one blue and the other red, on the pans and on the blackboard enables the user to identify their origin; it is not reproduced in this paper.

After analysis of the object type and of the nature (occurrence or set) of both operands, the system displays the appropriate operators on the scales screen. Scroll arrows are added to the scales if its screen cannot fit in all the operators. An operator is selected by clicking on it. After validating a condition and in order to prevent graphic congestion, the scales are cleared and an indicator is set on the variable(s) involved in the condition. 
The scales may have a tray containing weights that represent potential values for a given operand (Figure 8). So the user may take one or several weights from this tray and bring them to a pan. At the same time, it informs the user of available values and saves him from entering data with the keyboard.

Let query Q1 be: 'Find all the names of people who are less than 18 years old.' (Figure 6). The user first brings the icon of an example object (a person) to the blackboard. Attributes of this object are then displayed in panels linked to the icon and give a more detailed view of the object. The user states his comparison condition by taking the age attribute and bringing it to a pan of the scales. The second operand is defined by selecting the other pan in order to display the cursor and then by entering the value with the keyboard. After analysis of both operands, the system displays the numerical operators; the user chooses the ' $<$ ' operator. The condition is iconified and may be called back.
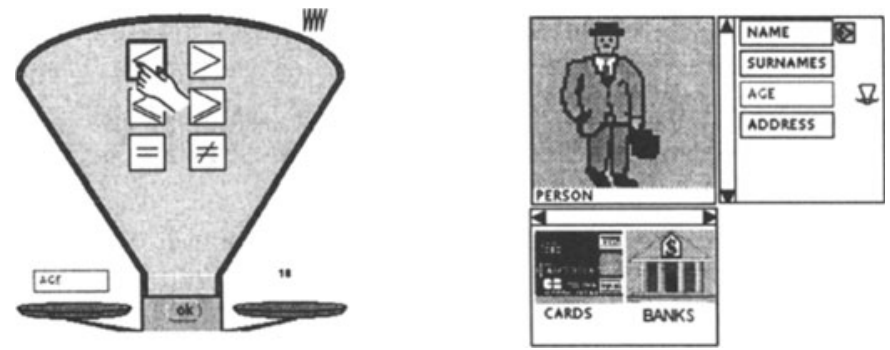

Figure 6 Query Q1, a comparison condition.

Stickers, with an eye drawn on them, permit the user to specify the operation commonly called projection in the database domain. The selection of attributes to be displayed in the result is performed by means of these stickers. In query Q1, a sticker is brought to the attribute name.

\subsection{The lasso metaphor}

\section{Basic features}

The goal of the lasso metaphor is to facilitate the expression of set operators such as set comparison and set combination operators. This metaphor is based on the notion of Venn Diagrams, already used but in a rudimentary way in some graphical languages (e.g., CANDID (Schneider and Trépied, 1989)).

The principles of this metaphor are:

- A lasso visualizes a set such as a class, a multivalued attribute or a set constant; this lasso is supposed to enclose all the objects belonging to this set.

- The lasso has a label connected to it and indicating the set.

- An object may be enclosed by several lassos, this is shown by an overlapping of these lassos. 
- By default, enclosed objects are not visualized. The zones delimited by the lassos are characterized by their color and intensity. A high intensity will indicate a non-empty zone (at least one object) whereas an average intensity will say nothing about the zone $(0,1$ or $\mathrm{N}$ objects) and a white color will indicate an empty zone (no object).

The graphic aspect of lassos may obviously be improved (like all the visual representations appearing in this paper). The fact that a lasso can be used to enclose several objects may seem surprising. In fact, this metaphor already exists in this form within environments designed for end users such as MacPaint and ClarisWorks.

The lasso is also used as a tool in order to switch from the object notion to the set notion. It enables the user, from an example object described on the blackboard, to obtain the set of objects verifying the same properties. These lassos are situated on the tool kit. To use them, the user takes a lasso, brings it to the blackboard and fills in its label by dragging on it the icon of an example object. Thus the lasso represents the set of objects verifying the same properties as the stated example object. This set may then be used in set comparisons or in set combination to describe an example object by showing its membership to certain classes.

\section{Comparisons involving sets}

Comparison of an occurrence to a set and comparison of two sets are said to be complex (Catarci and Santucci, 1995). Nevertheless, it is necessary to provide the user with a means of expressing such queries. Used within the scales, the lassos help to visualize the set comparison operators: membership, inclusion, intersection, etc. Each comparison operator has an iconic representation. Here, we detail comparisons of an occurrence to a set; comparisons of two sets follow the same approach.

Comparison of an occurrence to a set can be modeled in the form $\mathrm{x} \theta \mathrm{E}$ where:

- $\quad x$ is an occurrence (visualized with a bowl on the scales pans)

- E a set (visualized with a lasso)

- $\theta$ is an operator belonging to the following list:

$\theta 1=$ 'is_the_sole_object_of', called 'sole':

$(x \theta 1 \mathrm{E} \Leftrightarrow x \in \mathrm{E} \wedge$ $\theta y /(y \neq x \wedge y \in \mathrm{E}))$

$$
\begin{aligned}
\theta 2 & =\text { 'is_an_object_of, called 'in': } \quad(x \theta 2 \mathrm{E} \Leftrightarrow x \in \mathrm{E}) \\
\theta 3 & =\text { 'is_one_of_the_objects_of', called 'not sole': } \\
\qquad(x \theta 3 \mathrm{E} \Leftrightarrow x \in \mathrm{E} \wedge y y /(y \neq x \wedge y \in \mathrm{E})) & \Leftrightarrow \quad(x \theta 4 \mathrm{E} \Leftrightarrow x \notin \mathrm{E}) \\
\theta 4 & =\text { 'is_not_an_object_of', called 'out': }
\end{aligned}
$$

Membership $\left(\theta_{1}, \theta_{2}, \theta_{3}\right)$ and non-membership $\left(\theta_{4}\right)$ operators are visualized by means of icons on the scales screen. Each icon is an intuitive representation of its corresponding operator (Figure 7). The occurrence belongs to the set if the bowl is 
inside the lasso, the occurrence does not belong to the set if the bowl is outside the lasso.

In order to help the user distinguish the appropriate operator, the system makes a dichotomy between membership and non-membership operators and displays only two icons at the same time on the scales screen. The icon on the right-hand side of the scales screen visualizes the non-membership operator $\theta 4$. The membership operators may be reached with the icon on the left-hand side: the operator by default is $\theta 2$. The user may specify his condition more precisely by selecting the two other possible operators, $\theta_{1}$ and $\theta 3$, with the scroll arrows.
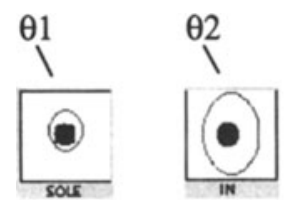

membership operators

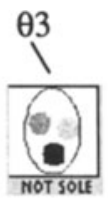
.

Figure 7 Set / occurrence comparison operators.

Let query Q2 be: 'Find all the people who have a blocked card.' (Figure 8). The user first details the attribute cards between person and card by selecting it. The set of cards associated with a person is then visualized with a lasso and the attributes of these cards are displayed. Next, he sets a comparison condition on the attribute states of cards by bringing it to a pan of the scales. The path from the type of the initial example object (i.e., person) is multivalued so the variable pointing out the attribute is seen as a set and is displayed with a lasso.
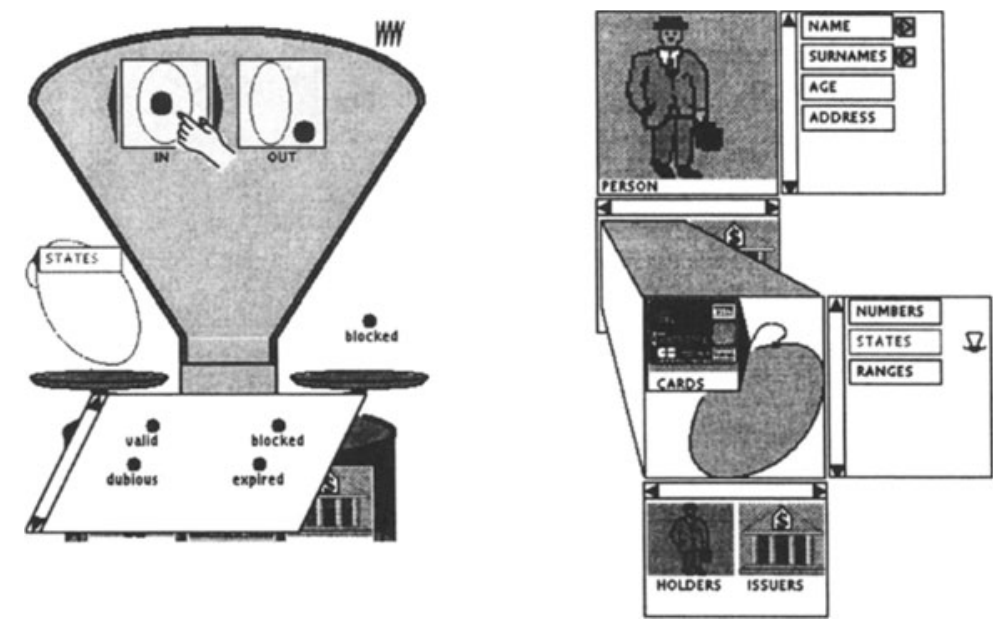

Figure 8 Query Q2, a set/occurrence comparison. 
The possible values of the attribute states are known in advance and are limited in number. Consequently, they are represented by weights (a bowl plus its label) and are placed on the tray in front of the scales. The second operand 'blocked' is brought from the tray to the empty pan; then the operators are displayed and the membership icon $\theta_{2}$ is selected.

These set comparison operators also answer the universal quantification problem. To our knowledge, they are no languages which propose a non-textual solution to the expression of universal quantification except the use of graphical conventions on Venn Diagrams (Schneider and Trépied, 1989) and of boxes (Whang and al., 1992).

Here, we take the example of the comparison of a set variable to an occurrence constant. The user shall be able to indicate if he wishes the condition to be verified for at most one occurrence of this set or for all of them.

Let query Q3 be: 'Find all the people who have all their cards blocked.' (Figure 9). It corresponds to the query Q2 with a universal quantification in the comparison condition. To indicate that all the occurrences of the set must have the value 'blocked', the user selects an operator on the scales screen. By means of the scroll arrows, he chooses the iconic representation that corresponds to the operator 'is_the_sole_object_of'. So, 'blocked' must be the only possible value of this set: $\forall x \in$ cards.states, $x=$ 'blocked', which answers the query.

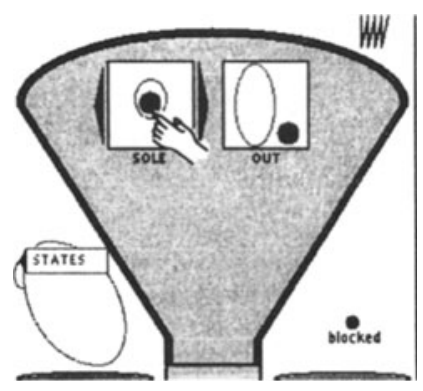

Figure 9 Query Q3, universal quantification.

\section{Class combination}

The lasso metaphor enables the user to formulate class combination operators such as conjunction, exclusive or inclusive disjunction and difference. Class to be combined are visualized with lassos. The expression of a combination is done by clicking on the corresponding zones inside the lassos. Using this method replaces the use of Boolean operators which, in spite of causing mistakes (Reisner, 1988), are still commonly employed in query languages to support conjunction or disjunction. In the following example, we show how a difference may be simply formulated with VISTA.

Let query Q4 be: 'Find the card numbers of all the non-Jackpot cards.' (Figure 10). The user first describes both example objects (card and Jackpot card) 
separately and then brings each icon to the label of a lasso in order to generate the corresponding classes. The arrangement of lassos (i.e., overlapping, inclusion and disjunction) is managed by the system. It follows covering constraints from the supertype by its subtypes and disjunction constraints of the subtypes. Difference is specified by selecting only the zone not surrounded by the lasso of the Jackpot cards (Figure 10a). After validating the combination, a new icon is generated (Figure 10b).

Difference between classes allows the user to express negation on sets whereas most non-textual languages do not support it. It enhances the expressive power of the language.

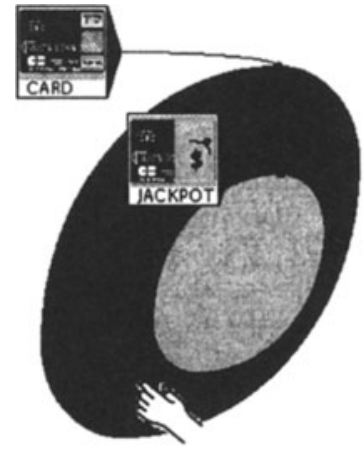

(a) combination

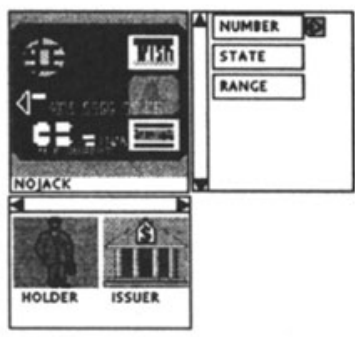

(b) generated icon

Figure 10 Query Q4, difference between classes.

\subsection{The shelf metaphor}

Visual languages inherently need space, and the limitations imposed by the screen may hinder or prevent the expression of some queries. So, mechanisms are suggested (e.g., several windows or a virtual space with scroll bars) but make query expression less easy. With the shelf metaphor, we suggest an innovative solution which is well integrated within the query room.

The shelf metaphor enables the user to construct complex queries by steps: he describes parts of queries, that we call subqueries, and composes them incrementally. It reduces the possible graphic congestion of a complex query and encourages breaking down a complex problem into several smaller problems.

The shelf is fastened to the wall above the tool kit. Stocking a subquery is done by bringing the icon of the example object from the blackboard to the shelf. Then this object is cleared from the blackboard and the user may describe another part of the query. The subquery is iconified according to the following rules:

- The image is that of the icon brought to the shelf. 
- The label is that of the icon brought to the shelf plus a serial number. This label may be customized: e.g. 'Minor' for a person who is less than 18 years old.

Let query Q5 be: 'Find all the banks who have clients that are minors.' (Figure 11). The query is split into two steps. In the first step, the user specifies the subquery 'minor person' (it corresponds to the query Q1) and stocks it on the shelf. In a second step, he states a condition between the clients of the bank (the right-hand side operand) and a minor (an object of the subquery is taken back from the shelf and placed on a pan).

Another way to express Q5 is to use a set comparison between the set of the clients of the bank (multivalued attribute) and the set of all the minors (class). As in many languages, a same query can be expressed in different ways.

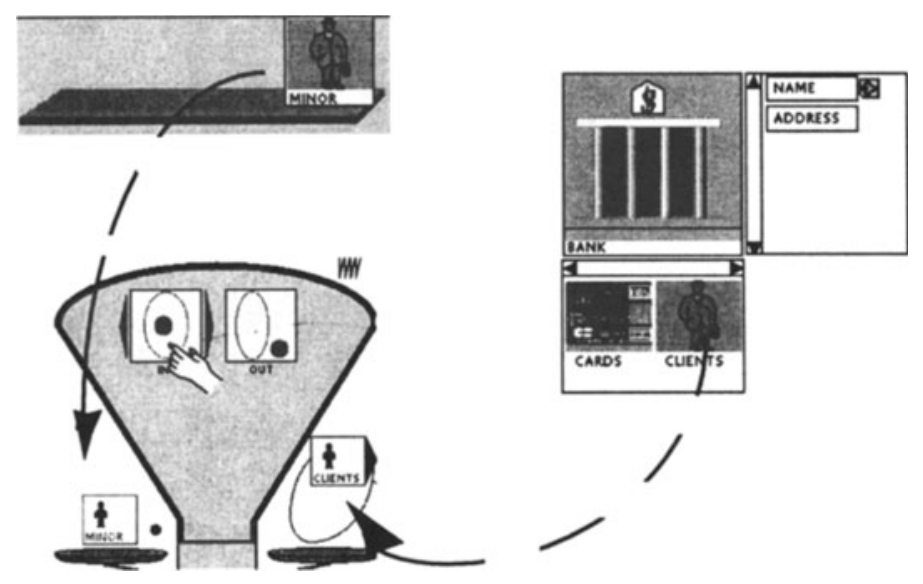

Figure 11 Query Q5, incremental query composition.

\subsection{Formalization and expressive power}

The syntax of the language is formalized with a BNF grammar which outline is given in appendix.

The expressive power of the language was evaluated. The chosen approach consisted in using a notion of completeness already defined on an algebra for complex objects (Bélières, 1997). To do so, we first established matches between the abstraction mechanisms of the underlying object model and the one proposed in VISTA. That enabled us to experiment with VISTA the operators of the algebra and to show that the expressive power of VISTA was close to the one of an algebra for complex objects. More details on this algebra and on the way of expressing its operators with VISTA are given in (Bélières, 1997). 


\section{THE SYSTEM ARCHITECTURE}

In this section we present a general overview of the system, shown in Figure 12. The main constraint was to use the existing relational database and its DBMS (Sybase Release 11).

The general architecture of the system is based on two layers:

- The DBMS interface supplies the communication with the relational DBMS.

- The end-user interface displays the semantic schema, controls the user manipulations and supports the display of the query result.

An elicitation of a semantic (VISTA) schema from the existing relational schema is required. This task is achieved by providing an internal representation of the VISTA schema.

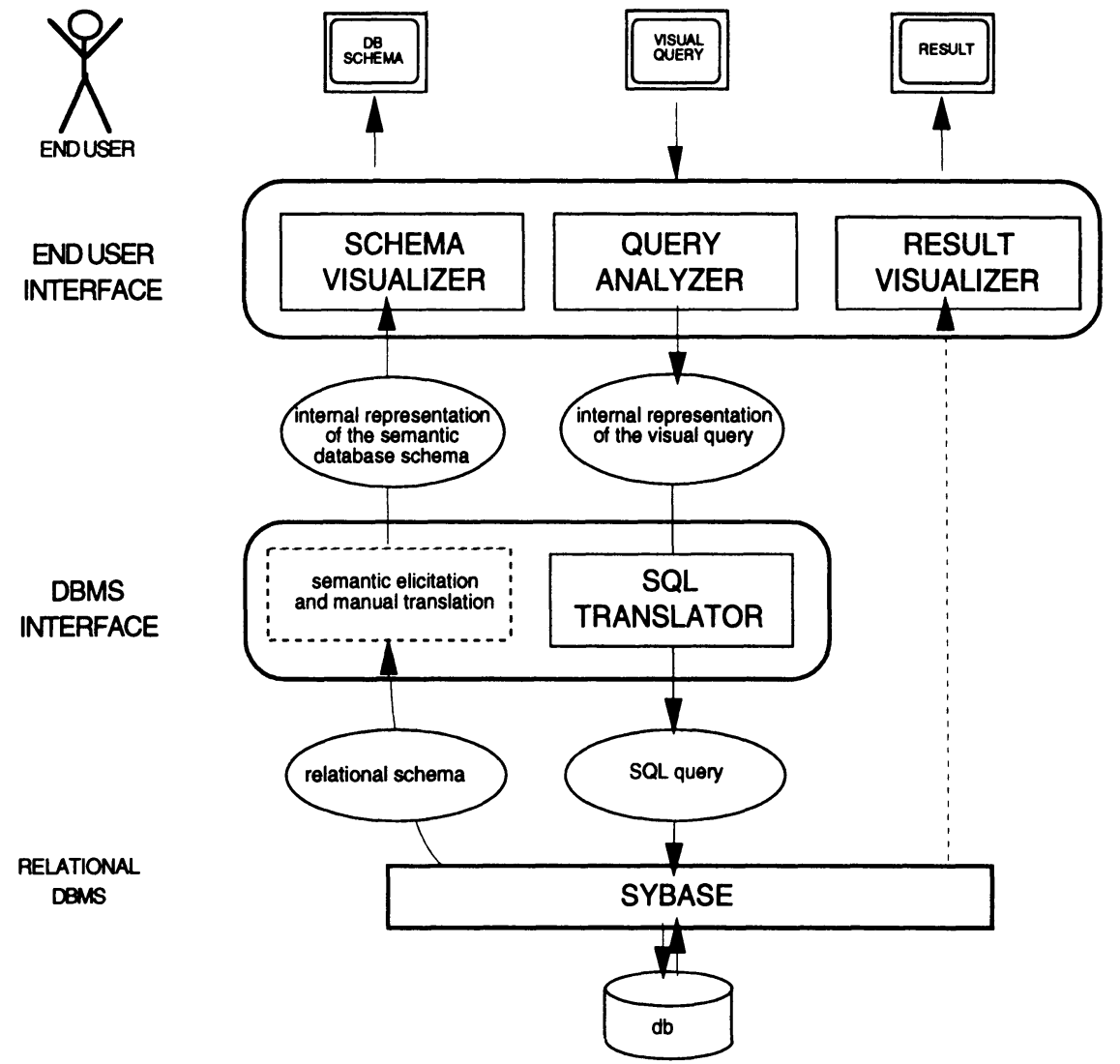

Figure 12 The system architecture.

Then the user may work with VISTA. Using the schema visualizer, the schema is displayed according to the requests of the user. This latter draws up his visual query and the query analyzer gives it an internal representation. The SQL 
translator produces an SQL query. The DBMS returns the result which is displayed with the result visualizer (however in the actual implementation the result is still in the tabular format of Sybase).

Several approaches have been given in the literature in order to set up a semantic schema from a relational schema (e.g., (Chiang and al., 1994) and (Premerlani and Blaha, 1994)). From this set of works, we have drawn up a synthetic list of transformation rules between the concepts found in a relational schema and the ones proposed in VISTA. However it can not be complete due to the variety of modeling within a same model and to the difference between the data model concepts. Moreover we were confronted with non 3NF relational schemas. It is the reason why we really do not believe in a purely mechanical approach for database reverse engineering.

\section{CONCLUSIONS}

The concept of metaphor appears to be a useful complement to the reverse engineering of relational databases as it may follow the semantic elicitation process. We use it to translate theoretical database concepts (i.e., the data model concepts and the language operators) into accessible and familiar ones for end users, allowing them to query an existing relational database.

We have presented VISTA, a visual database language, which is based on a generalized use of metaphors. The user is set in the concrete and familiar environment of a room. Query formulation consists of direct and natural manipulations on objects in this room. The scales metaphor guides the user while he specifies a condition. The lasso metaphor allows expressing set comparisons and combinations as well as universal quantification in an intuitive way. The shelf metaphor answers the problem of graphic congestion.

A prototype has been implemented in an industrial setting at the Call Center of the Sligos Company in a UNIX / X-Window / Sybase DBMS / C++ environment on SUN workstations. The aim of the VISTA prototype was to supply team managers with a tool for banking activity analysis. Typically they are experts in their field but they do not have any knowledge in computer science. VISTA has already been used for one year in this industrial setting. We began by a period of training. The basic mechanisms (building queries by defining selections and comparison conditions with stickers and the scales) were understood in a few hours. Then assistance was given to users in concrete situations (when somebody calls the Call Center for instance) to show how to transform their needs in VISTA queries. A complementary approach could consist in the generation of the textual paraphrase of the visual query; it would help the user check the consistency of his query. The VISTA users show satisfaction for this language that enables them to express the queries they need on the database independently of the technical team. Such a language may be placed at the end of the semantic elicitation process when we want the end user to exploit the database schema and to query the database.

All the illustrations of visual interaction presented in this paper are taken from actual interaction sessions. Another application we have studied is the broadcast 
shopping (in French,"Télé-achat"). Only new icons must be created for each object type of the new database schema. Future work involves visualization of the results and extension to deductive functionalities.

\section{Acknowledgements}

We are grateful to Bertrand Bricault, Paul Briot, Franck Gadin, Chi Hai Lim, Emmanuel Poiraux, Stéphane Tardy who have taken part in the development and implementation of the VISTA prototype and to the various end users in the Call Center for their helpful comments.

\section{REFERENCES}

Aufaure-Portier, M.A. and Trépied, C. (1996) A Survey of Query Languages for Geographic Information Systems, Proceedings of IDS-3 (3rd International Workshop on Interfaces to Databases), published in Springer Verlag's Electronic Workshops in Computing Series (URL : http://www.springer. co.uk/eWiC/Workshops/IDS3.html), Edinburgh, UK, 14 p.

Batini, C., Catarci, T., Costabile, M.F. and Levialdi, S. (1993) On Visual Representations for Database Query Systems. Proceedings of L'interface des mondes réels et virtuels, Montpellier, France, pp. 273-283.

Bélières, B. and Trépied, C. (1995) Un langage visuel de bases de données associé à un modèle sémantique à objets. Proceedings of Inforsid'95 (Informatique des organisations et systèmes d'information et de décision), Grenoble, France, pp. 393-412.

Bélières, B. (1997) VISTA : Un langage métaphorique et visuel pour l'interrogation de bases de données, $\mathrm{PhD}$ thesis, Tours, France, $220 \mathrm{p}$.

Boursier, P. and Mainguenaud, M. (1992) Spatial Query Languages : Extended SQL vs. Visual Languages vs. Hypermaps. Proceedings of the 5th International Conference on Spatial Data Handling, Charleston SC, USA, pp. 249-259.

Calcinelli, D. and Mainguenaud, M. (1994) Cigales, A Visual Query Language for Geographical Information System: the User Interface. Journal of Visual Languages and Computing, vol.5, pp. 113-132.

Catarci, T. and Santucci, G. (1995) Diagrammatic Vs Textual Query Languages: A Comparative Experiment. Proceedings of the third Working Conference on Visual Database Systems, Lausanne, Switzerland, pp. 57-74.

Chang, S.K., Costabile, M.F. and Levialdi, S. (1992) A Framework for Intelligent Visual Interface Design for Database Systems. Proceedings of the first International Workshop on Interfaces to Database Systems, Glasgow, United Kingdom, pp. 377-391.

Chiang, R.H.L., Barron, T.M. and Storey, V.C. (1994) Reverse Engineering of Relational Databases: Extraction of an EER model from a Relational Database. Data \& Knowledge Engineering, vol.12, pp.107-142.

Dennebouy, Y., Andersson, M., Auddino, A., Dupont, Y., Fontana, E., Gentile, M. and Spaccapietra, S. (1995) SUPER: Visual Interfaces for Object + 
Relationship Data Models. Journal of Visual Languages and Computing, vol.5, pp. 73-99.

Egenhofer, M.J. and Bruns, H.T. (1995) Visual Map Algebra: A DirectManipulation User Interface for GIS. Proceedings of the third Working Conference on Visual Database Systems, Lausanne, Switzerland, pp. 211-226. Fujii, H. and Korfhage, R.R. (1991) Features and a Model for Icon Morphological Transformation. Proceedings of IEEE Workshop on Visual Languages, Kobe, Japan, pp. 240-245.

Hull, R. and King, R. (1987) Semantic Database Modeling: Survey, Applications, and Research Issues. ACM Computing Surveys, vol.19, n³, pp. 201-260.

Mark, D.M. (1992) Spatial Metaphors for Human-Computer Interaction. Proceedings of the 5th International Conference on Spatial Data Handling, Charleston SC, USA, pp. 104-112.

Meyer, B. (1992) Beyond Icons: Towards New Metaphors for Visual Query Languages for Spatial Information Systems. Proceedings of the first International Workshop on Interfaces to Database Systems, Glasgow, United Kingdom, pp. 113-135.

Mohan, L. and Kashyap, R.L. (1993) A Visual Query Language for Graphical Interaction With Schema-Intensive Databases. IEEE Transactions on Knowledge and Data Engineering, vol.5, ${ }^{\circ} 5$, pp. 843-858.

Premerlani, W.J. and Blaha, M.R. (1994) An Approach for Reverse Engineering of Relational Databases. Communications of the ACM, Vol.37, ${ }^{\circ} 5$, pp. $42-49$.

Reisner, P. (1988) Query Languages. In 'Handbook of Human-Computer Interaction', Eds North-Holland, USA, pp. 257-280.

Roby-Brami, A. (1993) Direct Manipulation of Data. In 'Non-Visual HumanComputer Interactions', Eds Libbey Eurotext, pp. 79-96.

Schneider, M. and Trépied, C. (1989) Graphical Description and Query Interface for Databases, Proceedings of the 4th IFIP Working Conference on Engineering for Human-Computer Interaction, Napa Valley, California, USA, pp 63-84.

Staes, F., Tarantino, L. and Tiems, A. (1991) A Graphical Query Language for Object Oriented Databases. Proceedings of IEEE Workshop on Visual Languages, Kobe, Japan, pp. 205-210.

Trépied, C. (1995) Dynamic Icons for a Visual Spatio-Temporal Query Language. Proceedings of JEC-GI'95 (Joint European Conference on Geographical Information), The Hague, The Netherlands, pp. 84-89.

Vadaparty, K., Aslandogan, Y.A. and Ozsoyoglu, G. (1993) Towards a Unified Visual Database Access. Proceedings of SIGMOD'93 (Special Interest Group on Management of Data), Washington DC, USA, pp. 357-366.

Whang, K.Y., Malhotra, A., Sockut, G.H., Burns, L. and Choi, K.S. (1992) TwoDimensional Specification of Universal Quantification in a Graphical Database Query Language. IEEE Transactions on Software Engineering, vol.18, n³, pp. 216-224. 


\section{APPENDIX}

\section{Simplified VISTA syntax definition in BNF}

The complete BNF syntax is available in [Beli97].

$<$ VISTA_query $>::=\left(<\right.$ example_object $>[<\text { mark }>]_{1}^{N} \mid<$ marked_shelf_object $>$ $\left.[<\operatorname{mark}>]_{0}^{N}\right)[<\text { save_example_object }>]_{0}^{1}$

$<$ example_object $>::=<$ object $>[<\text { comparison_condition }>]_{0}^{\mathrm{N}}$

$<$ object $>::=(<$ cupboard_object $>\mid<$ hierarchy_object $>\mid<$ combi_object $>\mid$ $<$ shelf_object>)

$<$ save_example_object> ::= *save, in the database schema, the derived subtype defined by the query*

$<$ shelf_object> $::=(<$ non_marked_shelf_object $>\mid<$ marked_shelf_object $>)$

$<$ non_marked_shelf_object $>:=<$ example_object $>$

$<$ marked_shelf_object $>::=<$ example_object $>[<\operatorname{mark}>]_{1}^{\mathrm{N}}$

$<$ cupboard_object> ::=(<initial_object $>\mid<$ stored_object $>)$

$<$ initial_object> ::= *selection of an icon representing an AOT defined by the designer*

$<$ stored_object> ::= *selection of an icon representing a derived subtype stored in the schema*

$<$ hierarchy_object $>::=*$ selection of an icon from a classification board visualizing a certain spec/gen hierarchy*

$<$ combi_object $>::=<$ extract_example_object $><$ combination $>$

$<$ combination $>::=(<$ object_class $><$ combi_operator $><$ object_class $>)$

[<combi_operator $><$ object_class) $]_{0}^{1}$

$<$ object_class $>::=<$ lasso_on_object $><$ example_object $>$

$<$ extract_example_object> ::= *switch from the set notion to the object notion*

$<$ lasso_on_object> ::= *switch from the object notion to the set notion*

$<$ mark $>::=*$ mark an attribute to be displayed in the result*

$<$ comparison_condition $>::=(<$ occ/occ_compar $>\mid<0$ occ/set_compar $>\mid$ $<$ set/set_compar>)

$<$ occ/occ_compar $>::=<$ occurrence_expression $><$ occ/occ_compar_operator $>$ $<$ occurrence_expression>

$<$ occ/set_compar $>::=<$ occurrence_expression $><0 c c /$ set_compar_operator $>$ $<$ set_expression $>$

$<$ set/set_compar $>::=<$ set_expression $><$ set/set_compar_operator $><$ set_expression $>$ 


\section{BIOGRAPHY}

Bruno BELIERES received the Ph.D. degree in computer science from the University of Tours (France) in 1997. He is currently employed by CSC Financial Services Group in Paris as a Senior Project Engineer. He is working on various projects in the area of end user interfaces. His research interests include visual query languages and database reverse engineering.

Claude TREPIED received the Ph.D. degree in computer science from the University of Clermont-Ferrand II (France) in 1990. He is currently an assistant professor at the University of Tours and a researcher at the LISI (Laboratoire d'Ingénierie des Systèmes d'Information) of Lyon. His current research interests are in both theoretical and application-oriented aspects of visual formalisms for spatio-temporal database modeling and querying. 